\title{
Computing the Laplacian spectrum of linear octagonal-quadrilateral networks and its applications
}

\author{
Jia-Bao Liu ${ }^{1,2}$, Zhi-Yu Shi ${ }^{1}$, Ying-Hao Pan ${ }^{1}$, Jinde Cao ${ }^{2, *}$, M. Abdel-Aty ${ }^{3,4}$, Udai \\ Al-Juboori ${ }^{5}$ \\ ${ }^{1}$ School of Mathematics and Physics, Anhui Jianzhu University, Hefei 230601, China \\ ${ }^{2}$ School of Mathematics, Southeast University, Nanjing 210096, China \\ ${ }^{3}$ Center for Photonics and Smart Materials, Zewail City of Science and Technology, Egypt \\ ${ }^{4}$ Mathematics Department, Faculty of Sciences, Sohag University, Egypt \\ ${ }^{5}$ College of Arts and Science, Applied Science University, Kingdom of Bahrain
}

\begin{abstract}
Let $L_{n}$ denote linear octagonal-quadrilateral networks. In this paper, we aim to firstly investigate the Laplacian spectrum on the basis of Laplacian polynomial of $L_{n}$. Then, by applying the relationship between the coefficients and roots of the polynomials, the Kirchhoff index and the complexity are determined.
\end{abstract}

Keywords: Laplacian matrix; Resistance distance; Kirchhoff index; Complexity

AMS subject classification: 05C50, 05C90

\section{Introduction}

In this article, we only consider simple, undirected and connected graphs. Suppose $G=\left(V_{G}, E_{G}\right)$ is a graph with vertex set $V_{G}=\left\{v_{1}, v_{2}, \cdots, v_{n}\right\}$ and edge set $E_{G}=\left\{e_{1}, e_{2}, \cdots, e_{m}\right\}$. Let $D(G)=$ $\operatorname{diag}\left\{d_{1}, d_{2}, \cdots, d_{n}\right\}$ be a degree diagonal matrix, where $d_{i}$ is the degree of $v_{i}$ in $G$. The adjacency matrix $A(G)$ of $G$ is an $(0,1)$-matrix with order $n$. Then we can get the Laplacian matrix, which is defined as $L(G)=D(G)-A(G)$. Let $0=\mu_{1}<\mu_{2} \leq \cdots \leq \mu_{n}$ be the eigenvalues of $L(G)$. According to the characteristic polynomial of the matrix $L(G)$, we can get Laplacian spectrum of $L_{n}$ [5]. For more notations and terminologies, one can be referred to 6 .

At this point, some parameters are introduced. The distance, denoted by $d_{i j}$, is the length of a shortest path between nodes $i$ and $j$, which was named as Wiener index 3.13. This is well-known distance-based topological descriptor, that is

$$
W(G)=\sum_{i<j} d_{i j}
$$

In the electrical network theory, the resistance distance was firstly proposed by Klein and Randić [10]. According to this concept, we obtain the interpretation of physical community: the resistance distance between the nodes $i$ and $j$ of the graph $G$ is denoted by $r_{i j}$. One well-known resistance distance-based parameter called the Kirchhoff index 8,9$]$ is given by

$$
K f(G)=\sum_{i<j} r_{i j}
$$

The Kirchhoff index has attracted extensive attentions due to its wide applications in the fields of physics, chemistry and others. Despite all that, it is hard to deal with the Kirchhoff index of complex graphs. Thus, some researchers try to find some new techniques to compute the Kirchhoff index and

E-mail address: liujiabaoad@163.com, shizhiyuah@163.com, 17318533941@126.com, jdcao@seu.edu.cn, amisaty@gmail.com, udai.al-juboori@asu.edu.bh

* Corresponding author. 
obtain its formula. Given an $n$-vertex graph $G$, Klein and Lovász [4 16] proved independently that

$$
K f(G)=\sum_{\{u, v\} \subseteq V} r_{(G)}(u, v)=n \sum_{k=2}^{n} \frac{1}{\mu_{k}},
$$

where $0=\mu_{1}<\mu_{2} \leq \cdots \leq \mu_{n}(n \geq 2)$ are the eigenvalues of $L(G)$.

The number of spanning trees of the graph $G$, also known as the complexity of $G$, is the number of subgraphs that contain all the vertices of $G$ 2. In addition, all those subgraphs must be trees.

According to the decomposition theorem of Laplacian polynomial, Y. Yang et al., 2008 [14] obtained the Laplacian spectrum of linear hexagonal networks. J. Huang et al. [7] got the normalized Laplacian spectrum of linear hexagonal networks by using the decomposition theorem. Then, the Laplacian spectrum of linear phenylenes were derived [12]. Besides, Z. Zhu and J. Liu [17] obtained the Laplacian spectrum of generalized phenylenes. Thus, the extended considerations for calculating the Laplacian spectrum of linear octagonal-quadrilateral networks are shown in the following sections.

In the following, we introduce some theorems and notations in Section 2 . Then, we derive the Laplacian spectrum of $L_{n}$ by using the relationship between the coefficients and roots in Section 3 . An example of the result is given in Section 4 . The conclusion is summarized in Section 5.

\section{Preliminary}

First, we list some terminology, notations and some mature consequences in the following.

Given an $n \times n$ matrix, use $M\left[i_{1}, \cdots, i_{k}\right]$ to be a submatrix of $M$, which deletes the $i_{1}$-th, $\cdots, i_{k}$-th columns and rows. The characteristic polynomial of the matrix $M$ is denoted by $P_{M}(x)=\operatorname{det}(x I-M)$.

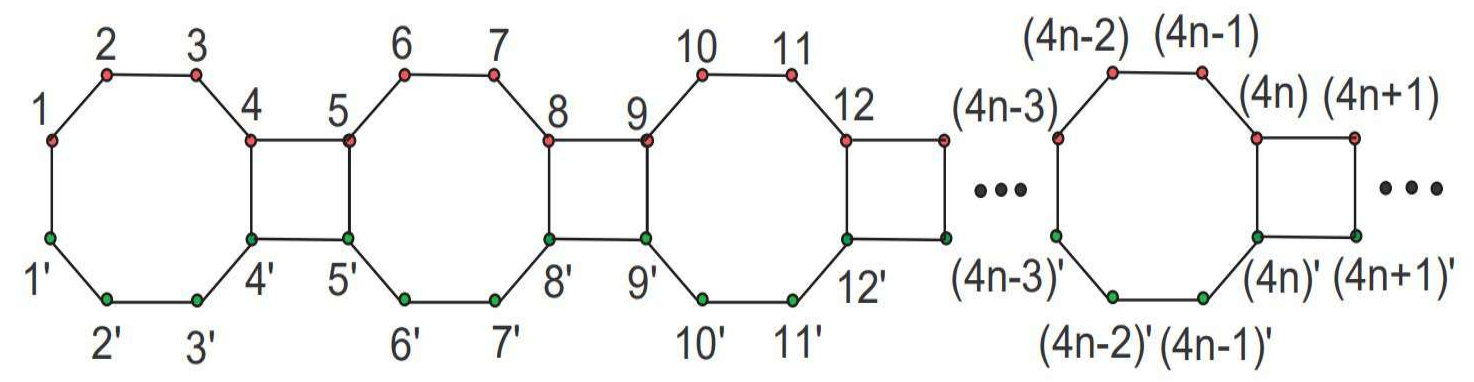

Figure 1: Linear octagonal-quadrilateral networks $L_{n}$.

With a suitable labelling of linear octagonal-quadrilateral networks as shown in Figure 1. Evidently, $\left|V\left(L_{n}\right)\right|=8 n+2,\left|E\left(L_{n}\right)\right|=10 n+1$. Obviously, $\pi=\left(1,1^{\prime}\right)\left(2,2^{\prime}\right) \cdots\left(4 n+1,(4 n+1)^{\prime}\right)$ is an automorphism of $L_{n}$. Set $V_{1}=\{1,2, \cdots, 4 n+1\}, V_{2}=\left\{1^{\prime}, 2^{\prime}, \cdots,(4 n+1)^{\prime}\right\}$.

Then $L\left(L_{n}\right)$ can be expressed by the following block matrices.

$$
L\left(L_{n}\right)=\left(\begin{array}{ll}
L_{V_{1} V_{1}} & L_{V_{1} V_{2}} \\
L_{V_{2} V_{1}} & L_{V_{2} V_{2}}
\end{array}\right)
$$

where

$$
L_{V_{1} V_{1}}=L_{V_{2} V_{2}}, L_{V_{1} V_{2}}=L_{V_{2} V_{1}}
$$

Let

$$
T=\left(\begin{array}{cc}
\frac{1}{\sqrt{2}} I_{4 n+1} & \frac{1}{\sqrt{2}} I_{4 n+1} \\
\frac{1}{\sqrt{2}} I_{4 n+1} & -\frac{1}{\sqrt{2}} I_{4 n+1}
\end{array}\right),
$$


then

$$
T L\left(L_{n}\right) T^{\prime}=\left(\begin{array}{cc}
L_{A} & 0 \\
0 & L_{S}
\end{array}\right)
$$

where $T^{\prime}$ is the transposition of $T$ and

$$
L_{A}=L_{V_{1} V_{1}}+L_{V_{1} V_{2}}, L_{S}=L_{V_{1} V_{1}}-L_{V_{1} V_{2}} .
$$

In the block matrix, we can easily get the decomposition theorem of Laplacian polynomial.

Theorem 2.1. 15 Assume that $L_{A}, L_{S}$ are defined as above. Then we can get

$$
P_{L\left(L_{n}\right)}(x)=P_{L_{A}}(x) P_{L_{S}}(x) .
$$

Theorem 2.2. [1] If $G$ is the path with $n$ vertices, the eigenvalues of $L(G)$ are $\nu_{i}, i=0,1, \cdots, n-1$.

$$
\nu_{i}=4 \sin ^{2}\left(\frac{\pi i}{2 n}\right) .
$$

Theorem 2.3. [2] If $G$ is a connected graph with $n$ vertices, where $\tau(G)$ is the complexity of $G$. Then

$$
\tau(G)=\frac{1}{n} \prod_{i=2}^{n} \mu_{i}
$$

Theorem 2.4. [11 (Matrix Tree Theorem) If $G$ is a connected graph with $n$ vertices and $L$ be the Laplacian matrix of $G$. Then the complexity of $G$ is

$$
\tau(G)=\operatorname{det}(L(i))
$$

where $i=1,2, \cdots, n$.

In the following, a flowchart is given according to the steps we have processed, which helps to understand the proposed approach. The explanations of these notations that appear in the flowchart are describes in Section 3 .

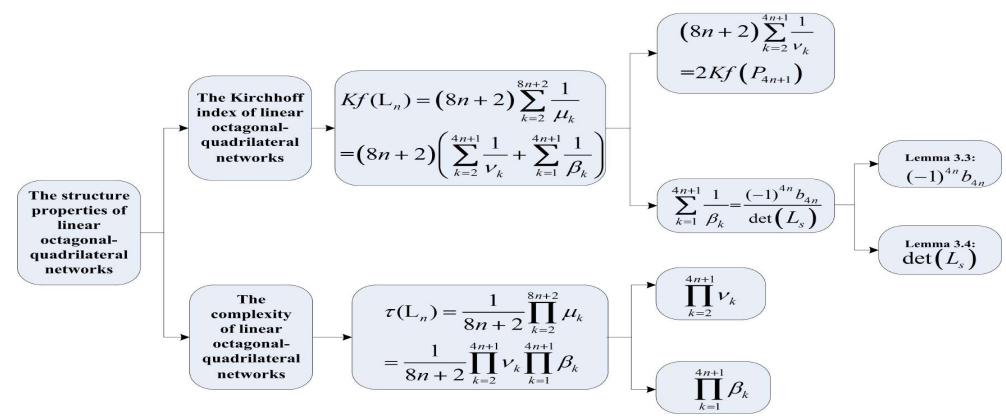

Figure 2: The steps processed in this paper. 


\section{Kirchhoff index and the complexity of $L_{n}$}

In this section, we get the general formula of the Kirchhoff index and the complexity. Using the decomposition theorem, we can get

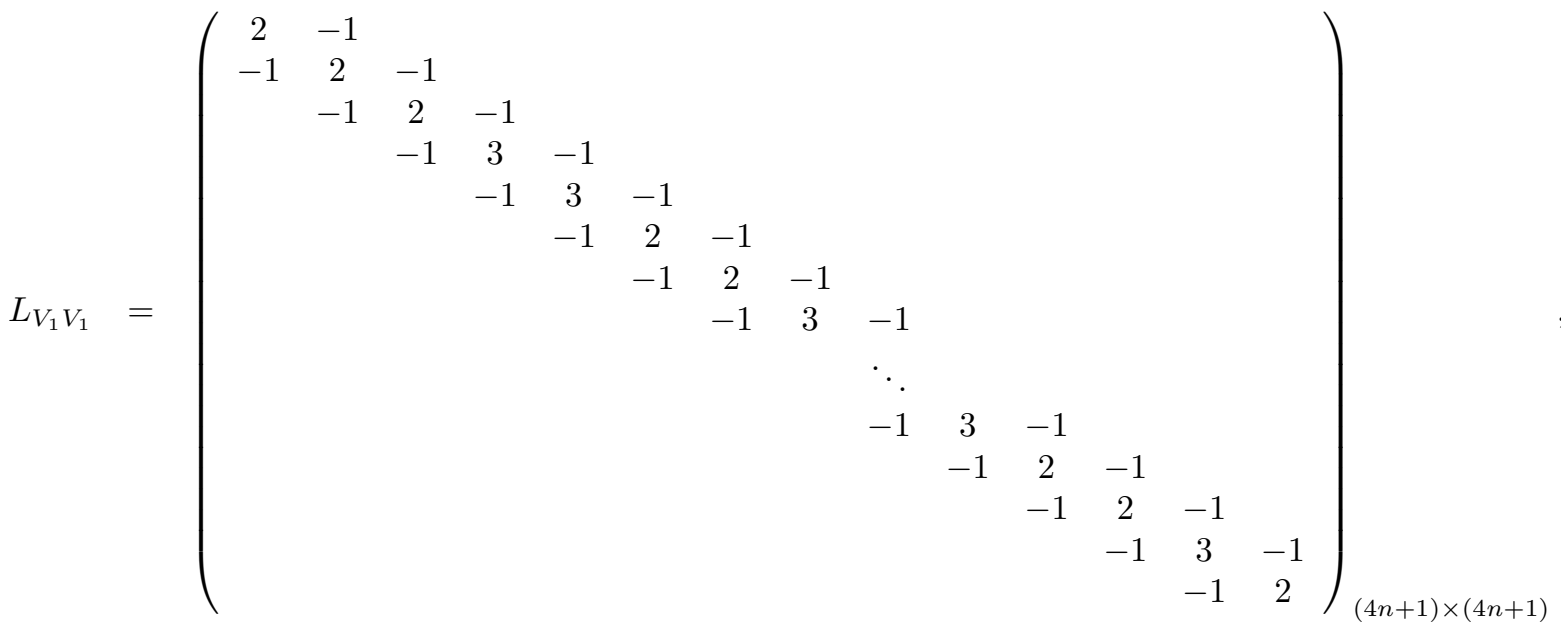

$$
\begin{aligned}
& L_{V_{1} V_{2}}=\operatorname{diag}\{-1,0,0-1,-1,0,0,-1, \cdots,-1,0,0,-1,-1\}_{(4 n+1)} .
\end{aligned}
$$

Through Theorem 2.1, one gets the Laplacian spectrum, which are composed of the eigenvalues of $L_{A}$ and $L_{S}$ of $L_{n}$ as follows.

$$
\left(\begin{array}{cccccccccc}
1 & -1 & & & & & & & & \\
-1 & 2 & -1 & & & & & & & \\
& -1 & 2 & -1 & & & & & & \\
& & -1 & 2 & -1 & & & & & \\
& & & -1 & 2 & -1 & & & & \\
& & & & & \ddots & & & & \\
& & & & & -1 & 2 & -1 & & \\
& & & & & & -1 & 2 & -1 & \\
& & & & & & & -1 & 2 & -1 \\
& & & & & & & & -1 & 1
\end{array}\right)_{(4 n+1) \times(4 n+1)}
$$




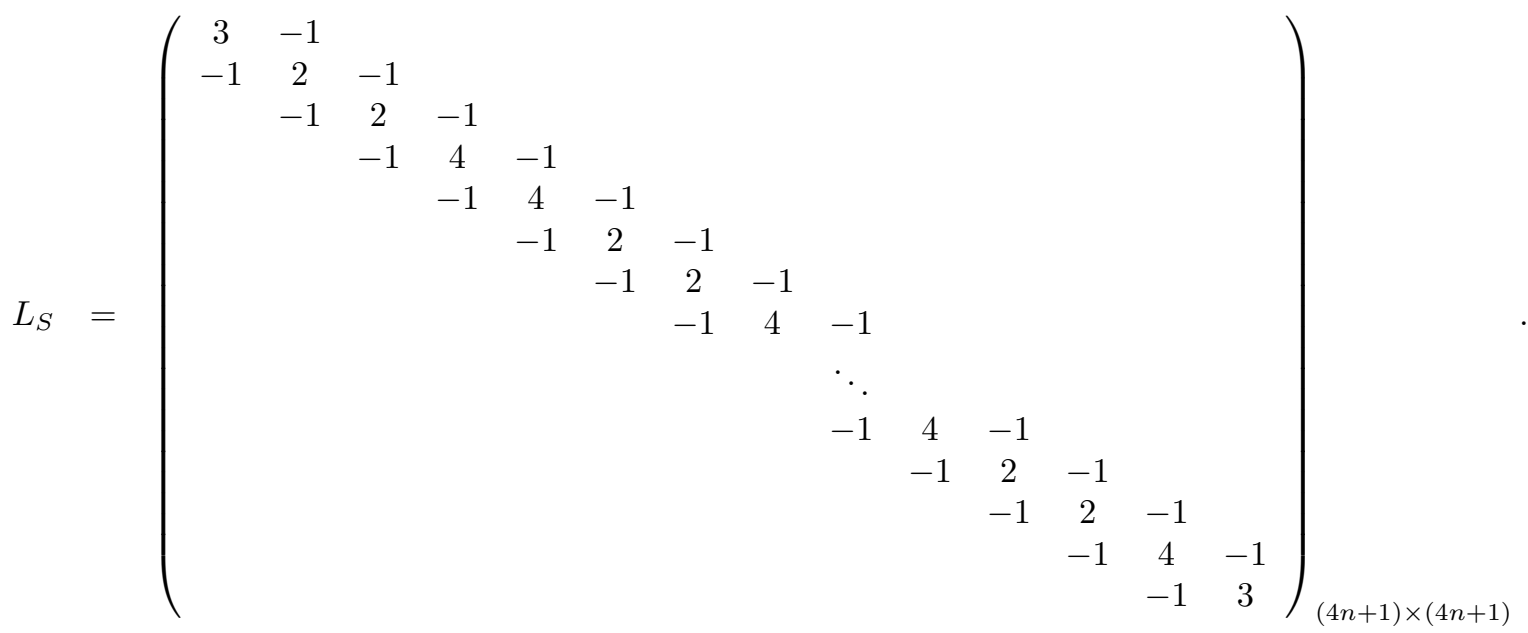

By calculation, we find that $L_{A}$ is obviously the Laplacian matrix of the path $P_{4 n+1}$.

Assume that $0=\nu_{1}<\nu_{2} \leq \nu_{3} \leq \cdots \leq \nu_{4 n+1}$ are the roots of polynomial $P_{L_{A}}(x)=0$, and $0<\beta_{1} \leq \beta_{2} \leq \beta_{3} \leq \cdots \leq \beta_{4 n+1}$ are the roots of polynomial $P_{L_{S}}(x)=0$. Therefore, we have

$$
K f\left(L_{n}\right)=(8 n+2)\left(\sum_{k=2}^{4 n+1} \frac{1}{\nu_{k}}+\sum_{k=1}^{4 n+1} \frac{1}{\beta_{k}}\right)=2 K f\left(P_{4 n+1}\right)+(8 n+2) \sum_{k=1}^{4 n+1} \frac{1}{\beta_{k}} .
$$

By applying the famous formula $K f\left(P_{n}\right)=\frac{n^{3}-n}{6}$, we can get

$$
2 K f\left(P_{4 n+1}\right)=\frac{8 n(4 n+1)(2 n+1)}{3} .
$$

Thus, we only need to calculate the latter part $\sum_{k=1}^{4 n+1} \frac{1}{\beta_{k}}$.

Let

$$
P_{L_{S}}(x)=\operatorname{det}\left(x I-L_{S}\right)=x^{4 n+1}+b_{1} x^{4 n}+\cdots+b_{4 n} x+b_{4 n+1} .
$$

By the relationship between coefficients and roots of the characteristic polynomial $P_{L_{S}}(x)$, we can get

$$
\sum_{k=1}^{4 n+1} \frac{1}{\beta_{k}}=\frac{(-1)^{4 n} b_{4 n}}{(-1)^{4 n+1} b_{4 n+1}}=\frac{(-1)^{4 n} b_{4 n}}{\operatorname{det}\left(L_{S}\right)} .
$$

Obviously, we obtain the $P_{L_{S}}(0)=(-1)^{4 n+1} \operatorname{det}\left(L_{S}\right)=b_{4 n+1}$ and the $(-1)^{4 n} b_{4 n}$ to be equal to sum of the principal minors of all $4 n$ columns and $4 n$ rows of $L_{S}$. Therefore, we make the $k$-th order principal submatrix to be $C_{k}$, which is composed of the first $k$ columns and rows of $L_{S}, k=1,2, \cdots, 4 n$. Let $c_{k}=\operatorname{det}\left(C_{k}\right)$. Then $c_{1}=3, c_{2}=5, c_{3}=7, c_{4}=23, c_{5}=85, c_{6}=147, c_{7}=209, c_{8}=689$ and for $i \geq 1$,

$$
\left\{\begin{array}{l}
c_{4 i+1}=4 c_{4 i}-c_{4 i-1} ; \\
c_{4 i+2}=2 c_{4 i+1}-c_{4 i} ; \\
c_{4 i+3}=2 c_{4 i+2}-c_{4 i+1} \\
c_{4 i+4}=4 c_{4 i+3}-c_{4 i+2} .
\end{array}\right.
$$

In the following, one gets the further terms of the above recurrence formulae.

$$
\left\{\begin{array}{l}
c_{4 i+1}=30 c_{4 i-3}-c_{4 i-7} ; \\
c_{4 i+2}=30 c_{4 i-2}-c_{4 i-6} ; \\
c_{4 i+3}=30 c_{4 i-1}-c_{4 i-5} ; \\
c_{4 i+4}=30 c_{4 i}-c_{4 i-4} .
\end{array}\right.
$$


For convenience, let $A$ and $B$ be $15+4 \sqrt{1} 4$ and $15-4 \sqrt{1} 4$. Throughout the rest of the context, we omit to introduce the definitions of $A$ and $B$, if there are no confusions.

Lemma 3.1. Let $c_{4 i+1(i \geq 0)}, c_{4 i+2(i \geq 0)}, c_{4 i+3(i \geq 0)}$ and $c_{4 i+4(i \geq 0)}$ be the sequences defined as above, for $0 \leq i \leq n-1$,

$$
\left\{\begin{array}{l}
c_{4 i+1}=\frac{(10+3 \sqrt{14}) A^{i}-(10-3 \sqrt{14}) B^{i}}{2 \sqrt{14}} ; \\
c_{4 i+2}=\frac{(18+5 \sqrt{14}) A^{i}-(18-5 \sqrt{14}) B^{i}}{2 \sqrt{14}} ; \\
c_{4 i+3}=\frac{(26+7 \sqrt{14}) A^{i}-(26-7 \sqrt{14}) B^{i}}{2 \sqrt{14}} ; \\
c_{4 i+4}=\frac{(86+23 \sqrt{14}) A^{i}-(86-23 \sqrt{14}) B^{i}}{2 \sqrt{14}} .
\end{array}\right.
$$

Now we make the $k$-th order principal submatrix to be $E_{k}$, which is composed of the last $k$ columns and rows of $L_{S}, k=1,2, \cdots, 4 n$. Let $e_{k}=\operatorname{det}\left(E_{k}\right)$. Then $e_{1}=3, e_{2}=11, e_{3}=19, e_{4}=27, e_{5}=89, e_{6}=$ $329, e_{7}=569, e_{8}=809$ and for $i \geq 1$,

$$
\left\{\begin{array}{l}
e_{4 i+1}=4 e_{4 i}-e_{4 i-1} \\
e_{4 i+2}=4 e_{4 i+1}-e_{4 i} \\
e_{4 i+3}=2 e_{4 i+2}-e_{4 i+1} \\
e_{4 i+4}=2 e_{4 i+3}-e_{4 i+2}
\end{array}\right.
$$

Then, we can get

$$
\left\{\begin{array}{l}
e_{4 i+1}=30 e_{4 i-3}-e_{4 i-7} \\
e_{4 i+2}=30 e_{4 i-2}-e_{4 i-6} \\
e_{4 i+3}=30 e_{4 i-1}-e_{4 i-5} \\
e_{4 i+4}=30 e_{4 i}-e_{4 i-4}
\end{array}\right.
$$

Using the above method, we can solve the infinite sequences $e_{4 i(i \geq 1)}\left(\right.$ resp. $e_{4 i+1(i \geq 0)}, e_{4 i+2(i \geq 0)}$, $\left.e_{4 i+3(i \geq 0)}\right)$ and get the general terms.

Lemma 3.2. Let $e_{4 i+1(i \geq 0)}, e_{4 i+2(i \geq 0)}, e_{4 i+3(i \geq 0)}$ and $e_{4 i+4(i \geq 0)}$ be the sequences defined as above, for $0 \leq i \leq n-1$,

$$
\left\{\begin{array}{l}
e_{4 i+1}=\frac{(11+3 \sqrt{14}) A^{i}-(11-3 \sqrt{14}) B^{i}}{2 \sqrt{14}} ; \\
e_{4 i+2}=\frac{(41+11 \sqrt{14}) A^{i}-(41-11 \sqrt{14}) B^{i}}{2 \sqrt{14}} ; \\
e_{4 i+3}=\frac{(71+19 \sqrt{14}) A^{i}-(71-19 \sqrt{14}) B^{i}}{2 \sqrt{14}} ; \\
e_{4 i+4}=\frac{(101+27 \sqrt{14}) A^{i}-(101-27 \sqrt{14}) B^{i}}{2 \sqrt{14}} .
\end{array}\right.
$$

Lemma 3.3. $(-1)^{4 n} b_{4 n}=\frac{1}{196}\left[(98+33 \sqrt{14}) A^{n}+(98-33 \sqrt{14}) B^{n}\right]+\frac{9 n}{14}\left[(4+\sqrt{14}) A^{n}+(4-\sqrt{14}) B^{n}\right]$.

Proof. Since numeric expression $(-1)^{4 n} b_{4 n}$ is equal to sum of the principal minors of all $4 n$ columns and $4 n$ rows of $L_{S}$, we can get 


$$
\begin{aligned}
(-1)^{4 n} b_{4 n}= & \sum_{i=1}^{4 n+1} \operatorname{det}\left(L_{S}[i]\right) \\
= & \sum_{k=0}^{n} \operatorname{det} L_{S}[4 k+1]+\sum_{k=0}^{n-1} \operatorname{det} L_{S}[4 k+2]+\sum_{k=0}^{n-1} \operatorname{det} L_{S}[4 k+3]+\sum_{k=0}^{n-1} \operatorname{det} L_{S}[4 k+4] \\
= & c_{4 n}+e_{4 n}+\sum_{k=1}^{n-1} c_{4 k} e_{4 n-4 k}+\sum_{k=0}^{n-1} c_{4 k+1} e_{4 n-4 k-1}+\sum_{k=0}^{n-1} c_{4 k+2} e_{4 n-4 k-2}+\sum_{k=0}^{n-1} c_{4 k+3} e_{4 n-4 k-3} \\
= & c_{4 n}+e_{4 n}+\sum_{k=1}^{n-1} c_{4 k} e_{4(n-k)}+\sum_{k=0}^{n-1} c_{4 k+1} e_{4(n-k-1)+3}+\sum_{k=0}^{n} c_{4 k+2} e_{4(n-k-1)+2} \\
& +\sum_{k=0}^{n-1} c_{4 k+3} e_{4(n-k-1)+1} .
\end{aligned}
$$

For convenience, we set

$$
\left\{\begin{array}{l}
D_{1}=\sum_{k=1}^{n-1} c_{4 k} e_{4(n-k)} \\
D_{2}=\sum_{k=0}^{n-1} c_{4 k+1} e_{4(n-k-1)+3} \\
D_{3}=\sum_{k=0}^{n-1} c_{4 k+2} e_{4(n-k-1)+2} \\
D_{4}=\sum_{k=0}^{n-1} c_{4 k+3} e_{4(n-k-1)+1}
\end{array}\right.
$$

Then

$$
\begin{aligned}
D_{1} & =\sum_{k=1}^{n-1} c_{4 k} e_{4(n-k)} \\
& =\sum_{k=1}^{n-1}\left[\frac{(86+23 \sqrt{14}) A^{k-1}-(86-23 \sqrt{14}) B^{k-1}}{2 \sqrt{14}} \cdot \frac{(101+27 \sqrt{14}) A^{n-k-1}-(101-27 \sqrt{14}) B^{n-k-1}}{2 \sqrt{14}}\right] \\
& =\frac{n-1}{56}\left[(17380+4645 \sqrt{14}) A^{n-2}+(17380-4645 \sqrt{14}) B^{n-2}\right]+\frac{\sqrt{14}}{392}\left(A^{n-1}-B^{n-1}\right) . \\
D_{2} & =\sum_{k=0}^{n-1} c_{4 k+1} e_{4(n-k-1)+3} \\
& =\sum_{k=0}^{n-1}\left[\frac{(10+3 \sqrt{14}) A^{k}-(10-3 \sqrt{14}) B^{k}}{2 \sqrt{14}} \cdot \frac{(71+19 \sqrt{14}) A^{n-k-1}-(71-19 \sqrt{14}) B^{n-k-1}}{2 \sqrt{14}}\right] \\
& =\frac{n}{56}\left[(1508+403 \sqrt{14}) A^{n-1}+(1508-403 \sqrt{14}) B^{n-1}\right]+\frac{11 \sqrt{14}}{392}\left(A^{n}-B^{n}\right) .
\end{aligned}
$$




$$
\begin{aligned}
D_{3} & =\sum_{k=0}^{n-1} c_{4 k+2} e_{4(n-k-1)+2} \\
& =\sum_{k=0}^{n-1}\left[\frac{(18+5 \sqrt{14}) A^{k}-(18-5 \sqrt{14}) B^{k}}{2 \sqrt{14}} \cdot \frac{(41+11 \sqrt{14}) A^{n-k-1}-(41-11 \sqrt{14}) B^{n-k-1}}{2 \sqrt{14}}\right] \\
& =\frac{n}{56}\left[(1508+403 \sqrt{14}) A^{n-1}+(1508-403 \sqrt{14}) B^{n-1}\right]+\frac{4 \sqrt{14}}{392}\left(A^{n}-B^{n}\right) . \\
D_{4} & =\sum_{k=0}^{n-1} c_{4 k+3} e_{4(n-k-1)+1} \\
& =\sum_{k=0}^{n-1}\left[\frac{(26+7 \sqrt{14}) A^{k}-(26-7 \sqrt{14}) B^{k}}{2 \sqrt{14}} \cdot \frac{(11+3 \sqrt{14}) A^{n-k-1}-(11-3 \sqrt{14}) B^{n-k-1}}{2 \sqrt{14}}\right] \\
& =\frac{n}{56}\left[(580+155 \sqrt{14}) A^{n-1}+(580-155 \sqrt{14}) B^{n-1}\right]+\frac{\sqrt{14}}{392}\left(A^{n}-B^{n}\right) .
\end{aligned}
$$

Therefore, we can have $(-1)^{4 n} b_{4 n}=c_{4 n}+e_{4 n}+D_{1}+D_{2}+D_{3}+D_{4}$. Then

$$
(-1)^{4 n} b_{4 n}=\frac{1}{196}\left[(98+33 \sqrt{14}) A^{n}+(98-33 \sqrt{14}) B^{n}\right]+\frac{9 n}{14}\left[(4+\sqrt{14}) A^{n}+(4-\sqrt{14}) B^{n}\right] .
$$

This completes the proof.

Lemma 3.4. $\operatorname{det}\left(L_{S}\right)=\frac{(116+31 \sqrt{14}) A^{n-1}-(116-31 \sqrt{14}) B^{n-1}}{\sqrt{14}}$.

Proof. By expanding $L_{s}$ with regard to the last row, we have

$$
\begin{aligned}
\operatorname{det}\left(L_{S}\right) & =3 c_{4 n}-c_{4(n-1)+3} \\
& =\frac{3\left[(86+23 \sqrt{14}) A^{n-1}-(86-23 \sqrt{14}) B^{n-1}\right]}{2 \sqrt{14}}-\frac{\left[(26+7 \sqrt{14}) A^{n-1}-(26-7 \sqrt{14}) B^{n-1}\right]}{2 \sqrt{14}} \\
& =\frac{(116+31 \sqrt{14}) A^{n-1}-(116-31 \sqrt{14}) B^{n-1}}{\sqrt{14}} .
\end{aligned}
$$

This completes the proof.

Together with formulas (3.7) - (3.9) and lemmas 3.3 - 3.4, one can get the following theorem.

Theorem 3.5. For linear octagonal-quadrilateral networks $L_{n}$,

$$
K f\left(L_{n}\right)=\frac{8 n(4 n+1)(2 n+1)}{3}+\frac{(8 n+2)(-1)^{4 n} b_{4 n}}{\operatorname{det} L_{S}},
$$

where

$$
\begin{aligned}
(-1)^{4 n} b_{4 n} & =\frac{1}{196}\left[(98+33 \sqrt{14}) A^{n}+(98-33 \sqrt{14}) B^{n}\right]+\frac{9 n}{14}\left[(4+\sqrt{14}) A^{n}+(4-\sqrt{14}) B^{n}\right], \\
\operatorname{det} L_{S} & =\frac{(116+31 \sqrt{14}) A^{n-1}-(116-31 \sqrt{14}) B^{n-1}}{\sqrt{14}} .
\end{aligned}
$$

The Kirchhoff indices of $L_{n}$ are shown in Table 1, in which $n$ is from 1 to 15 .

The explicit formula for the complexity of $L_{n}$ is in the following. 
Table 1: Kirchhoff indices from $L_{1}$ to $L_{15}$

\begin{tabular}{c|c|c|c|c|c|c|c|c|c}
\hline$G$ & $K f(G)$ & $G$ & $K f(G)$ & $G$ & $K f(G)$ & $G$ & $K f(G)$ & $G$ & $K f(G)$ \\
\hline$L_{1}$ & 69.52 & $L_{4}$ & 1977.70 & $L_{7}$ & 9128.25 & $L_{10}$ & 24977.17 & $L_{13}$ & 52980.46 \\
$L_{2}$ & 336.42 & $L_{5}$ & 3608.06 & $L_{8}$ & 13274.07 & $L_{11}$ & 32790.45 & $L_{14}$ & 65613.20 \\
$L_{3}$ & 929.82 & $L_{6}$ & 5948.91 & $L_{9}$ & 18514.38 & $L_{12}$ & 42082.21 & $L_{15}$ & 80108.42 \\
\hline
\end{tabular}

Theorem 3.6. For octagonal-quadrilateral networks $L_{n}$,

$$
\tau\left(L_{n}\right)=\frac{4^{4 n}\left[(116+31 \sqrt{14}) A^{n-1}-(116-31 \sqrt{14}) B^{n-1}\right]}{\sqrt{14}(8 n+2)} \prod_{i=2}^{4 n+1} \sin ^{2}\left(\frac{\pi(k-1)}{8 n+2}\right) .
$$

Proof. According to Theorem 2.2, we get the eigenvalues of $L_{A}$, which are $\nu_{k}=4 \sin ^{2}\left(\frac{\pi(k-1)}{8 n+2}\right)(k=$ $1,2, \cdots, 4 n+1)$.

Together with Lemma 3.4 and Theorem 2.3, we know that

$$
\begin{aligned}
\tau\left(L_{n}\right) & =\frac{1}{8 n+2} \prod_{k=2}^{4 n+1} \nu_{k} \cdot \prod_{k=1}^{4 n+1} \beta_{k} \\
& =\frac{\operatorname{det}\left(L_{s}\right)}{8 n+2} \prod_{k=2}^{4 n+1} 4 \sin ^{2}\left(\frac{\pi(k-1)}{8 n+2}\right) \\
& =\frac{4^{4 n}\left[(116+31 \sqrt{14}) A^{n-1}-(116-31 \sqrt{14}) B^{n-1}\right]}{\sqrt{14}(8 n+2)} \prod_{k=2}^{4 n+1} \sin ^{2}\left(\frac{\pi(k-1)}{8 n+2}\right) .
\end{aligned}
$$

This completes the proof.

The complexity of $L_{n}$ is shown Table 2, in which $n$ is from 1 to 12 .

Table 2: The complexity from $L_{1}$ to $L_{12}$

\begin{tabular}{c|c|c|c|c|c|c|c}
\hline$G$ & $\tau(G)$ & $G$ & $\tau(G)$ & $G$ & $\tau(G)$ & $G$ & $\tau(G)$ \\
\hline$L_{1}$ & 31 & $L_{4}$ & 834241 & $L_{7}$ & 22449425279 & $L_{10}$ & 604114033423649 \\
$L_{2}$ & 929 & $L_{5}$ & 24999391 & $L_{8}$ & 672733610881 & $L_{11}$ & 18103261443808319 \\
$L_{3}$ & 27839 & $L_{6}$ & 749147489 & $L_{9}$ & 20159558901151 & $L_{12}$ & 542493729280825921 \\
\hline
\end{tabular}

\section{Example}

For example, we calculate the Kirchhoff index and the complexity for $L_{1}$. First, we use the decomposition theorem to obtain the block matrix. Then, the relationship between the coefficients and roots derives the explicit formulas for the Kirchhoff index and the complexity. Thus, we can obtain $L_{V_{1} V_{1}}$ and $L_{V_{2} V_{2}}$, as follows.

$$
L_{V_{1} V_{1}}=\left(\begin{array}{ccccc}
2 & -1 & & & \\
-1 & 2 & -1 & & \\
& -1 & 2 & -1 & \\
& & -1 & 3 & -1 \\
& & & -1 & 2
\end{array}\right)
$$




$$
L_{V_{2} V_{2}}=\left(\begin{array}{ccccc}
-1 & & & & \\
& 0 & & & \\
& & 0 & & \\
& & & -1 & \\
& & & & -1
\end{array}\right) \text {. }
$$

According to the decomposition theorem, one gets the two special matrices, $L_{A}$ and $L_{S}$ with order 5 , as follows

$$
\begin{aligned}
L_{A} & =\left(\begin{array}{ccccc}
1 & -1 & & & \\
-1 & 2 & -1 & & \\
& -1 & 2 & -1 & \\
& & -1 & 2 & -1 \\
& & & -1 & 1
\end{array}\right), \\
L_{S} & =\left(\begin{array}{ccccc}
3 & -1 & & & \\
-1 & 2 & -1 & & \\
& -1 & 2 & -1 & \\
& & -1 & 4 & -1 \\
& & & -1 & 3
\end{array}\right) .
\end{aligned}
$$

Obviously, $L_{A}$ is the Laplacian matrix of path $P_{5}$. Let $0=\nu_{1}<\nu_{2} \leq \nu_{3} \leq \nu_{4} \leq \nu_{5}$ be the roots of characteristic polynomial $P_{L_{A}}(x)=0$, and $0<\beta_{1} \leq \beta_{2} \leq \beta_{3} \leq \beta_{4} \leq \beta_{5}$ be the roots of characteristic polynomial $P_{L_{S}}(x)=0$.

Thus, we can obtain

$$
\begin{gathered}
K f(G)=2 K f\left(P_{5}\right)+10 \sum_{k=1}^{5} \frac{1}{\beta_{k}}=\frac{2155}{31} \\
\tau(G)=\frac{1}{10} \prod_{k=2}^{5} \nu_{k} \prod_{k=1}^{5} \beta_{k}=31 .
\end{gathered}
$$

Remark. According to Theorem 2.4, we can calculate the complexity of $L_{1}$. Meanwhile, it is found that the complexity is equal to that result which has been calculated by Laplacian spectrum.

\section{Conclusion}

We mainly use the decomposition theorem of Laplacian polynomial. The relationship between coefficients and roots of the polynomial is a necessary method for us to arrive the Kirchhoff index and the complexity of octagonal-quadrilateral networks. In addition, this method can be applied to other graphs.

\section{Author contribution}

Funding acquisition, J.-B Liu; Methodology, J.-B Liu, Jinde Cao; Formal analysis, J.-B Liu, Zhi-Yu Shi; Data curation, Writing-original draft, Zhi-Yu Shi, Ying-Hao Pan, M. Abdel-Aty, Udai Al-Juboori. 


\section{Funding}

The work was partly supported by China Postdoctoral Science Foundation (No. 2017M621579), Postdoctoral Science Foundation of Jiangsu Province (No. 1701081B) and Project of Anhui Jianzhu University (No. 2016QD116, 2017dc03 and 2017QD20).

\section{References}

[1] W.N. Anderson, T.D. Morely, Eigenvalues of the Laplacian of a graph, Linear and Multilinear Algebra 18 (1985) 141-145.

[2] F.R.K. Chung, Spectral Graph Theory, American Mathematical Society Providence, RI, 1997.

[3] A. Dobrynin, Branchings in trees and the calculation of the Wiener index of a tree, Match Communications in Mathematical and in Computer Chemistry 41 (2000) 119-134.

[4] I. Gutman, B. Mohar, The quasi-Wiener and the Kirchhoff indices coincide, Journal of Chemical Information and Modeling 36 (1996) 982-985.

[5] C. He, S.C. Li, W. Luo, L. Sun, Calculating the normalized Laplacian spectrum and the number of spanning trees of linear pentagonal chains, Journal of Computational and Applied Mathematics 344 (2018) 381-393.

[6] J. Huang, S.C. Li, The normalized Laplacians on both $k$-triangle graph and $k$-quadrilateral graph with their applications, Applied Mathematics and Computation 320 (2018) 213-225.

[7] J. Huang, S.C. Li, L. Sun, The normalized Laplacians degree-Kirchhoff index and the spanning trees of linear hexagonal chains, Discrete Applied Mathematics 207 (2016) 67-79.

[8] D.J. Klein, Resistance-distance sum rules, Croatica Chemica Acta 75 (2002) 633-649.

[9] D.J. Klein, O. Ivanciuc, Graph cyclicity, excess conductance, and resistance deficit, Journal of Mathematical Chemistry 30 (2001) 271-287.

[10] D.J. Klein, M. Randić, Resistance distances, Journal of Mathematical Chemistry 12 (1993) 81-95.

[11] K.H. Monfared, S. Mallik, An analog of Matrix Tree Theorem for signless Laplacians, Linear Algebra and its Applications 560 (2019) 43-55.

[12] Y.J. Peng, S.C. Li, On the kirchhoff index and the number of spanning trees of linear phenylenes, Match Communications in Mathematical and in Computer Chemistry 77 (2017) 765-780.

[13] H. Wiener, Structural determination of paraffin boiling points, Journal of the American Chemical Society 69 (1947) $17-20$.

[14] Y.J. Yang, H.P. Zhang, Kirchhoff Index of Linear Hexagonal Chains, International Journal of Quantum Chemistry 108(3) (2008) 503-512.

[15] Y.L. Yang, T.Y. Yu, Graph theory of viscoelasticities for polymers with starshaped, multiple-ring and cyclic multiple-ring molecules, Macromolecular Chemistry and Physics 186 (1985) 609-631.

[16] H.Y. Zhu, D.J. Klein, I. Lukovits, Extensions of the Wiener number, Journal of Chemical Information and Modeling 36 (1996) 420-428.

[17] Z.X. Zhu, J.-B. Liu, The normalized Laplacian, degree-Kirchhoff index and the spanning tree numbers of generalized phenylenes, Discrete Applied Mathematics 254 (2019) 256-267. 